\title{
Numerical analysis of crack propagation in a pull-out test
}

\author{
Jakub Gontarz ${ }^{1, *}$, and Jerzy Podgórski ${ }^{1}$ \\ ${ }^{1}$ Lublin University of Technology, Faculty of Civil Engineering and Architecture, Nadbystrzycka 40, 20-618 Lublin, Poland
}

\begin{abstract}
This paper describes the computer analysis of the pull-out test for determining the force needed to pull out a rock fragment, and the shape of this fractured fragment. The material analysed is sandstone. The analysis included a comparison of the various crack propagation methods in computer program using the Finite Element Method. The work also contains a description of performed laboratory tests and analytical considerations.
\end{abstract}

\section{Introduction}

The authors of this paper have analysed the pull-out test in which the Hilti anchor is pulled out of the sandstone rock. These anchors are normally used to anchor various construction elements. The described test is intended for the opposite purpose - to pull out the anchor together with a part of the rock. This is a different approach, because the anchor is designed not to destroy the material in which it is mounted, but to destroy itself. These analyses are intended to be used in mining, including mine rescue, where it is not possible to destroy rocks with explosives, which is a popular method. Preset undercut Hilti HDA-P M20×250 anchors with anchoring length of $25 \mathrm{~cm}$ will be used [1]. The picture of this anchor is shown in fig. 1 .

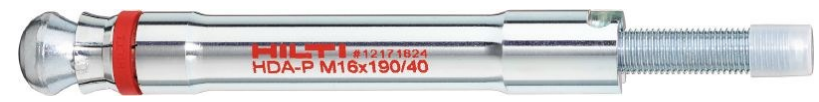

Fig. 1. Pre-set undercut Hilti HDA-P anchor.

To mount this anchor, it is placed in a prepared hole in the surface to be anchored. Then, a drill is attached to the anchor, and while drilling, the anchor undercuts itself with deflexed elements (fig. 2). fixing the anchor therefore consists in the contact between the material and the undercut, not the anchor side. So, the contact area is relatively small.

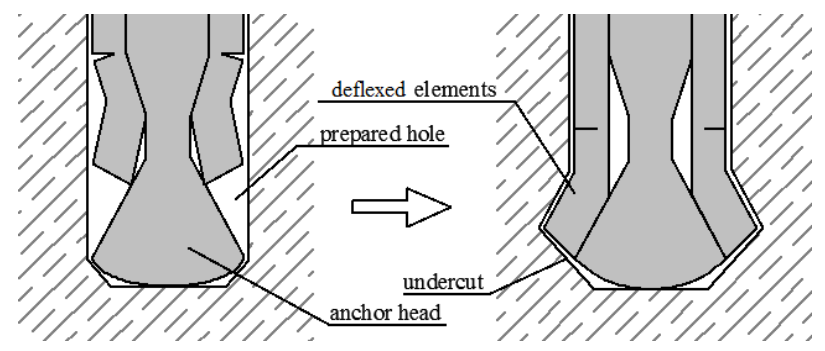

Fig. 2. Scheme of mounting the anchor.
The authors made an attempt to estimate the critical force and determine the size of a pulled-out rock fragment with X-FEM computer method of crack modelling in program Abaqus. Analyses focused on sandstone obtained from quarry in Braciszów in Poland. The main purpose of this article is to compare the results obtained for different methods of applying the load in computer program.

Similar problem is described in the article [2], but instead of undercut anchors, steel bars fixed inside surface layers of the examined material are used. Thus, the characteristic of the pull-out test is completely different from those described in the above paper.

In other articles [3] the capacity of adhesive anchors was examined. Also, in article [4] the fixed anchors surrounded by plastic pipe were investigated. In these tests the characteristic is similar to the one described in this work, but here the anchor was loaded cyclically.

\section{Description of the model}

Abaqus computer program was used for calculations. The X-FEM method was used for modelling the crack. Since this was an axially symmetrical task, it was modelled in 2D layout with axis of symmetry. The computational models is presented in fig. 3, where $h$ is the length of the anchor. The boundary conditions were modelled on the right and bottom edge. The size of the model was assumed to be large enough so that the boundary conditions did not affect the result.

The sandstone material was modelled as linearelastic with Young modulus $E=14.276 \mathrm{GPa}$, Poisson's ratio $v=0.247$, tensile strength $f_{t}=7.74 \mathrm{MPa}$, and the critical strain energy release rate $G_{I c}=0.355 \mathrm{~N} / \mathrm{mm}$. Laboratory tests from which these parameters were obtained are described in the further subsection. Tensile strength is also the stress that initiates the crack.

The load was simulated by several different methods. In the first method, there was no anchor in the model, but the load was simulated by the vertical displacement applied at the anchor undercut (see fig. 3). This method was divided into two variants. First - with horizontal

\footnotetext{
${ }^{*}$ Corresponding author: j.gontarz@pollub.pl
} 
blocking on the anchor edge, second - without this blocking. In the next method, the anchor was in the model. The load was simulated by vertical displacement applied to the upper edge of the anchor, and the contact between the anchor and sandstone existed, with three different friction coefficients: $0.01,0.2$ and 1 . There was no possibility to determine the exact friction coefficient between these two materials because in this test, rock is crushed under a heavy load, but still this crushed part transfers the load further.

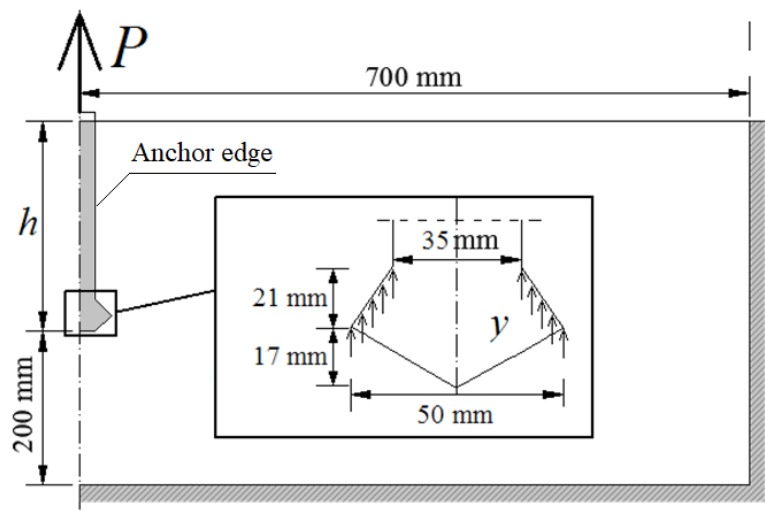

Fig. 3. Scheme of the task.

The critical force was determined by sum of the vertical reactions in nodes with applied displacement.

It was attempted to analytically estimate the critical force. For this purpose, the task was simplified to this shown in fig. 4.

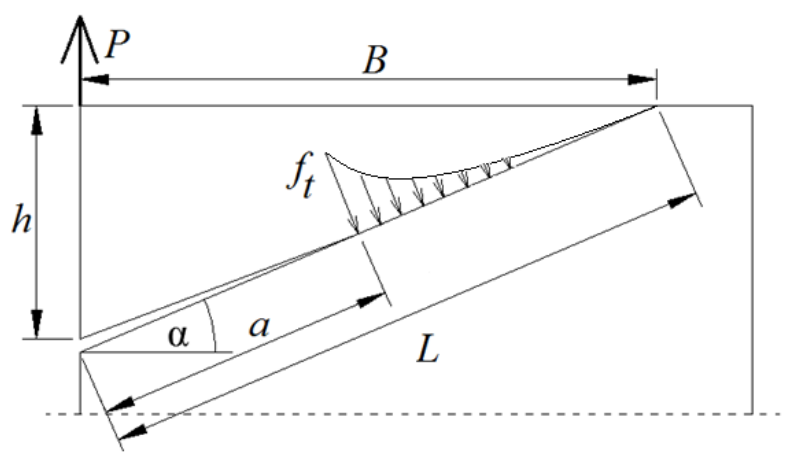

Fig. 4. Scheme for estimating the critical force $P$.

The shape of the stress that appears after the crack tip was simplified to a parabola. The shape of the parabola was assumed from Abaqus program from this test. The maximal principal stress was read on between the crack tip and the possible crack end. For different steps of calculation (different crack length $a$ ) it turned out that the stress field was similar for these steps. The longer the crack was, the narrower the parabola, yet proportional to the length $(L-a)$. The authors adjusted the coordinates of the parabola to the obtained plots, as it can be seen in fig. 5. The values greater than $0.3(L-a)$ were omitted, because the stress values are negligibly small.

The force $P$ was calculated from equilibrium of vertical forces. $P$ was equal to vertical component of stress field volume, which was a solid of revolution received from rotation of the stress field shown in fig. 4 . The volume of a solid of revolution was the product of area of a cross section (the parabola) and a circumference of a circle with a radius equal to the distance between the centre of rotation from the centre of gravity of the parabola:

$P(a)=2 \cdot \pi \cdot \cos (\alpha) \cdot c(a) \cdot A(a)$

where $c(a)$ is the function of the length between the vertical axis of symmetry and the parable centre gravity, $A(a)$ is the parable area. The angle of the crack is $\alpha=21^{\circ}$, and it was adopted as a mean from computer tests and tests on actual rock described in the following subsections.

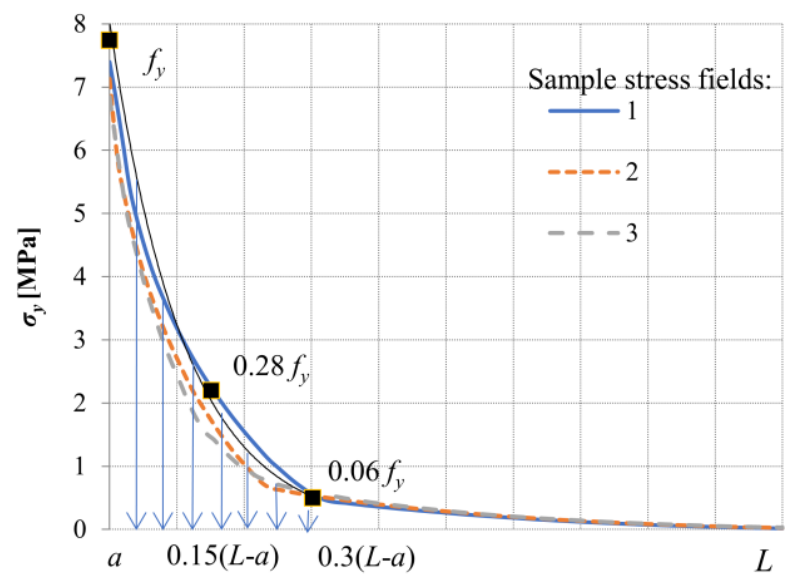

Fig. 5. Stress fields obtained for different crack length and a parabola that simplifies these stresses.

The graph showing the function $P(a)$ is presented in fig. 6. The maximum force was $62.928 \mathrm{kN}$, and it was obtained around in the middle of the crack path.

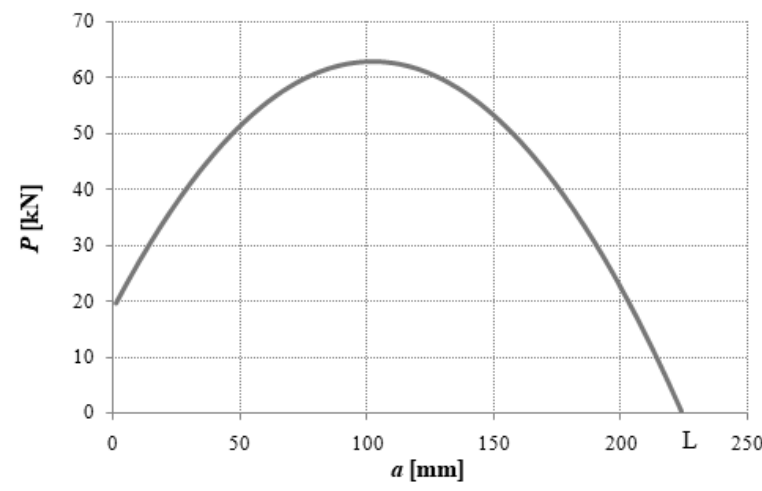

Fig. 6. Dependence between the pulling-out force and the anchor length in analytical method.

\section{Material parameters}

Correct modelling in the program requires determination of material parameters. A series of laboratory tests were performed on samples of the selected material.

\subsection{Compression test}

14 cubes of $8 \times 8 \times 8 \mathrm{~cm}$ dimensions were used to determine few material parameters. They were used to calculate the Young modulus and Poisson's ratio thanks to compression with extensometer tests. Then the 
compressive strength was obtained from the destructive compression test performed on the same samples.

Pictures from these tests are shown in fig. 7. On the left side there is visible a displacement sensor which measured the vertical deformations, and on the right side there is the extensometer that measured the horizontal deformations. It is mounted on steel plates glued to the opposite sides of samples.

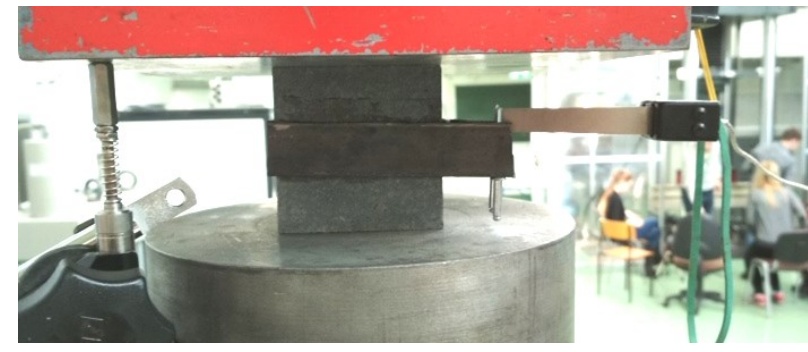

Fig. 7. Compression with extensometer test.

The Young modulus was calculated from the below equation:

$E=\frac{h \cdot \kappa}{A}$

where $h$ is the height of the sample, $A$ is the area of horizontal cross-section of the sample and $\kappa$ is the slope of the curve of compressive force to vertical deformations dependence.

Poisson's ratio was estimated from the dependency curve of horizontal deformations from the extensometer to vertical deformations from the displacement sensor. The compressive strength was obtained from the standard method as the ratio of the destructive force to the area of horizontal cross-section of the sample.

\subsection{Beam bending test}

The authors performed a three-point bending test on notched beams to calculate the stress intensity factor in mode I, and then, the critical strain energy release rate in mode $\mathrm{I}$.

Critical stress intensity factor is a material characteristic. It specifies the amount of stress concentration at the crack tip. There are three main modes of cracks. In the case of the problem described in this article, the most appropriate mode is mode I, which occurs when an opening of a crack is caused by tensile force perpendicular to the crack.

There are several methods for calculating this coefficient [5]. The authors of this article made threepoint bending tests on the discussed material. This test was performed on samples cut out in the middle to cause the specimen to crack half in size. Figure 8 shows how this test works, and fig. 9 shows the photo taken during the laboratory test.

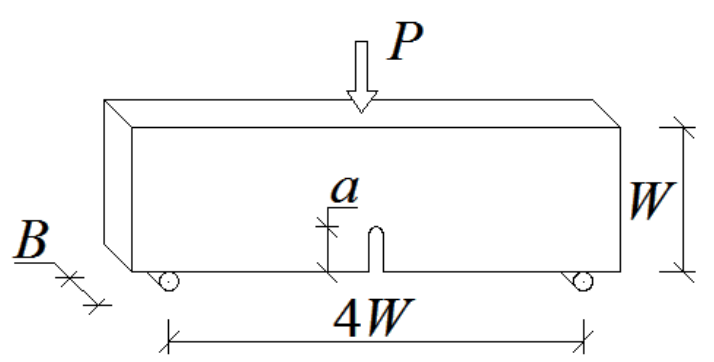

Fig. 8. Scheme of the three-point bending test with notches.

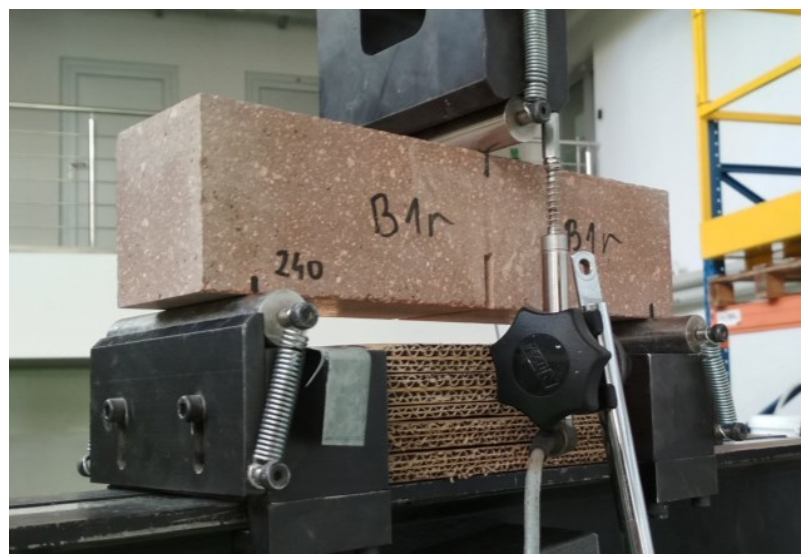

Fig. 9. Three-point bending test for notched beams.

There are several methods for calculating the stress intensity factor in mode I from the results obtained from the described test $[6,7]$. The authors of this article used the Bower equation described in [6]:

$K_{I c}=\frac{4 \cdot P}{B} \cdot \sqrt{\frac{\pi}{W}} \cdot\left[\begin{array}{l}1.6 \cdot\left(\frac{a}{W}\right)^{\frac{1}{2}}-2.6 \cdot\left(\frac{a}{W}\right)^{\frac{3}{2}}+ \\ 12.3 \cdot\left(\frac{a}{W}\right)^{\frac{5}{2}}-21.2 \cdot\left(\frac{a}{W}\right)^{\frac{7}{2}}+21.8 \cdot\left(\frac{a}{W}\right)^{\frac{9}{2}}\end{array}\right]$

$P$ used in this equation is the force that initiated the crack in this bending test, $a$ is the length of the notch, $W$ - the height of the beam, $B$ - the width of the beam. 6 samples were used for this test. The calculated stress intensity factor was $71.203 \mathrm{~N} / \mathrm{mm}^{3 / 2}$, which was close to factors from similar materials.

The critical strain energy release rate in mode I was calculated from equation [7]:

$$
G_{I c}=\frac{K_{I c}^{2}}{E}
$$

\subsection{Quasi-Brazilian test}

The authors also made a quasi-Brazilian test on cubes. Typically tests of traction during splitting are performed on cylindrical samples, but they are hard to obtain from such material, so the Abaqus program was used to find the field of stresses and then the tensile strength for cubic samples, the tensile strength was calculated thanks to the previous article of the author [8].

Figure 10 presents the samples after exemplary tests. Here heterogeneity of the examined material can be seen. 


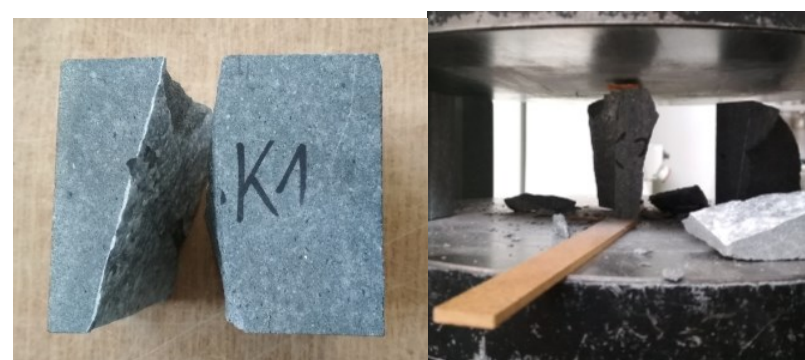

Fig. 10. The quasi-Brazilian test.

\section{Computer simulations}

The above material parameters were used to model the test in Abaqus for an $9 \mathrm{~cm}$ anchoring. The authors used the X-FEM method of crack propagation. Extended Finite Element Method is a method of simulating a fracture in the Finite Element Method, which is independent of the mesh. Modification of the shape function of element allows the finite element to be separated anywhere [9], so the mesh does not need to be fine. In the above example, the simplest criterion for crack initiation was chosen, which is, when the tensile stress exceeds the value of the tensile strength [10].

As it was described before, there are 5 methods of applying the pulling-out force:

- Without anchor, locked horizontal displacement,

- Without anchor, with horizontal displacement,

- With anchor, friction coeff. $=0.01$

- With anchor, friction coeff. $=0.2$,

- With anchor, friction coeff. $=1$,

Below, there are some exemplary figures from simulations from Abaqus. In fig. 11 there is one of the models before applying the load. The view of the damaged model from sample simulation is shown in fig. 12.

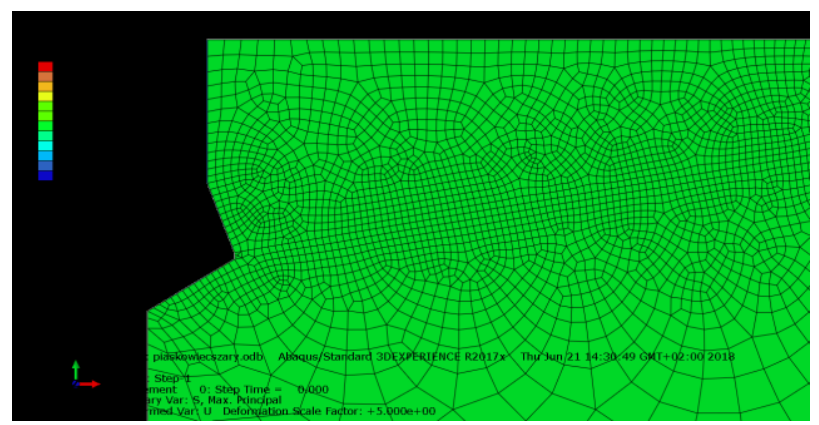

Fig. 11. Model of the test before applying the load.

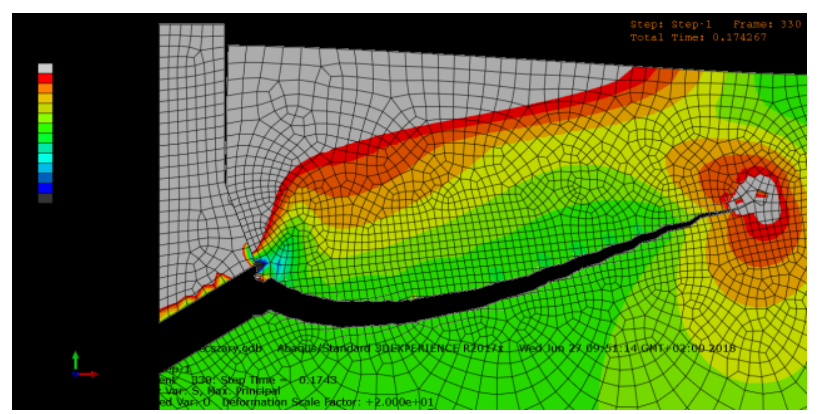

Fig. 12. Last step of calculation from simulation with anchor and friction coefficient $=0.2$.
As it can be seen, the crack started to propagate as expected, but the crack near the top edge began to distort and return. This is related to the limitations of X-FEM. The state of stress here was so complex that the program probably could not decide where to lead the crack. For various program settings and different mesh, it was not possible to cause the crack to go through to the end. The pulling-out force at the beginning grew and then decreased. Figure 13 is the graph showing how the force was changing during the crack growing in simulations. As it can be seen, there is very big difference between the results. The maximum force varies from about $100 \mathrm{kN}$ to $220 \mathrm{kN}$. It means that the model and the friction coefficient should be chosen very carefully, but this is difficult due to the fact that the material crushes in contact with the anchor. Also, the maximum force was obtained in different places, with different crack lengths.

In fig. 14 crack paths for different methods are shown. Here the results are also very different. As it will be proved in the next chapter, the shape closest to reality is the one for the friction coefficient 0.2 . It can be seen that the path of the crack at the very end behaves unnaturally in all models.

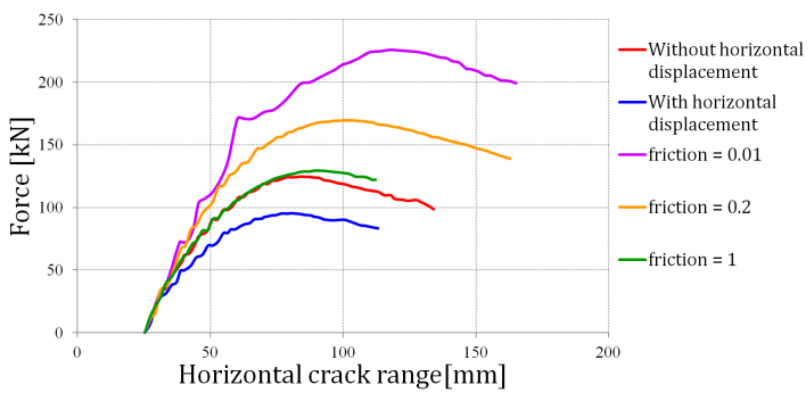

Fig. 13. Graph of force dependence on the horizontal range of the crack.

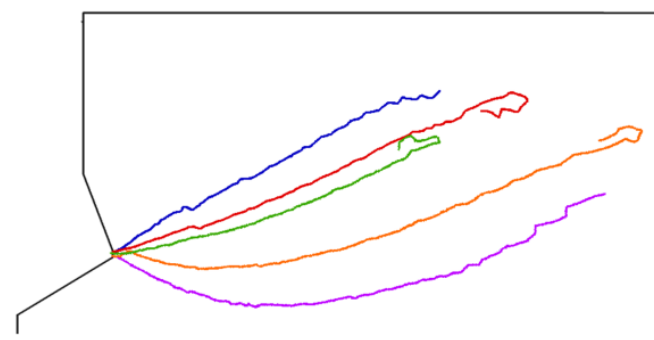

Fig. 14. Crack paths for different methods of applying the load. Colour scheme is the same as in fig. 13.

The main conclusion from the above analyses is that anchors should not be omitted in the analyses, however different friction coefficients have a very big influence on the result, both on the maximum force and the crack path.

\section{Pull-out test on actual rock}

Tests in the quarry were also made on the same stone and for the same depth of the anchor (fig. 15). For three successful tests, the average pulling-out force is $162 \mathrm{kN}$. Inspection of the damaged rock in fig. 16 allowed to state that the shape of the broken fragment was similar to that in the computer simulation, especially for the simulation with the anchor and with friction coefficient 0.2. 


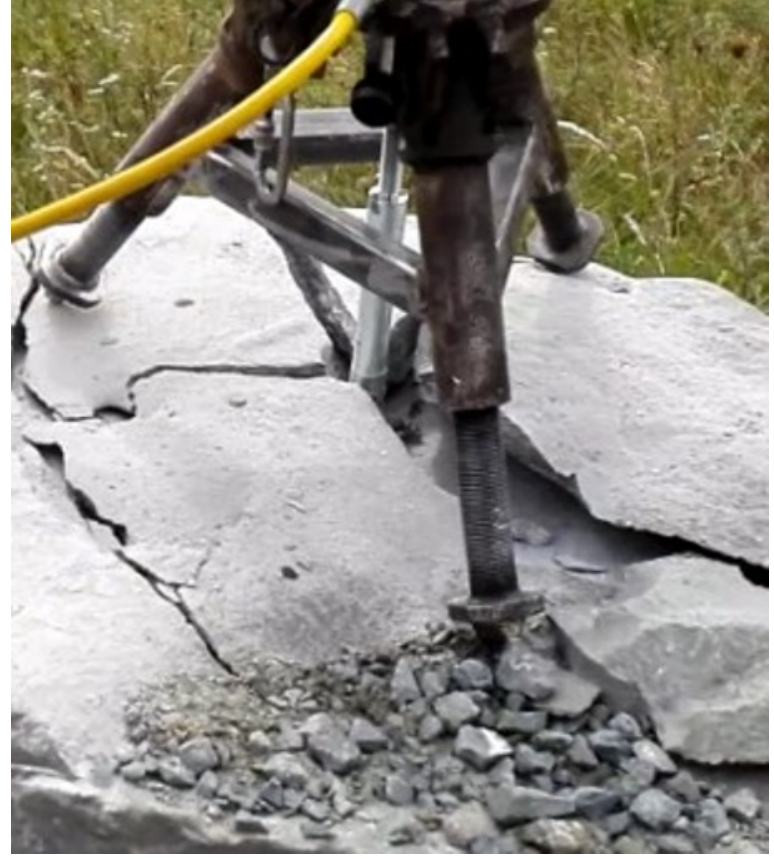

Fig. 15. Pull-out test performed on actual rock [11].*

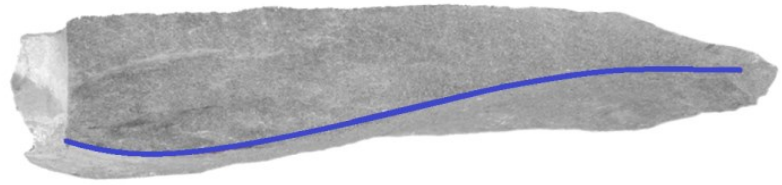

Fig. 16. A cross-section of the actual pull-out sandstone sample.*

Also, examining one of the pulled-out cones in fig. 17 it was possible to explain incorrect crack behaviour in computer simulations. It is evident that the width of the pulled-out fragment is different on the circumference. In most places, the rock broke perpendicular to the surface at the very end. Probably in simulations there should also be such a crack, but the Abaqus program had difficulty in simulating the forking of the crack.

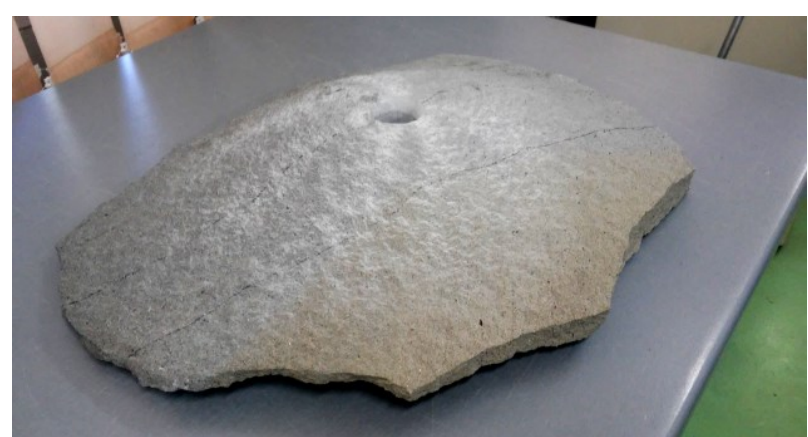

Fig. 17. The shape of the actual pull-out sandstone sample.*

\section{Summary}

Here are all the maximum force values for different methods that have been achieved:

- Analytical: $62.93 \mathrm{kN}$,

- Abaqus - locked horizontal displacement: $124.52 \mathrm{kN}$,

- Abaqus - with horizontal displacement: $95.31 \mathrm{kN}$,
- Abaqus - friction coeff. $=0.01: 225.90 \mathrm{kN}$,

- Abaqus - friction coeff. $=0.2: 169.40 \mathrm{kN}$,

- Abaqus - friction coeff. $=1: 129.25 \mathrm{kN}$,

- Mean from the actual test: $162.00 \mathrm{kN}$.

Unfortunately, the analytical method gave results completely incompatible with the others, so it should be improved, for example, by considering the forces parallel to the crack.

Also, as it was stated before, the results without the anchor are incorrect in relation to models with an anchor. The best obtained result is for simulation with anchor and for friction coefficient between the anchor and the rock $=0.2$, both in terms of the maximum obtained force, and the shape of the crack. However, it is not known if this coefficient is correct, especially since in fact this coefficient should be variable due to the changes of the rock during the test.

Computer simulations cannot be performed to the total breakage of the rock fragment, because before the end the crack begins to behave inconsistently to the test in reality. Fortunately, the maximum force was obtained before the occurrence of this phenomenon, which means that even if this phenomenon continues to occur, it is possible to determine the maximum force.

Research carried out as part of the project RODEST nr 2015/19/B/ST10/02817 financed by the Polish National Science Center.

*The pictures come from field research in the Braciszów mine.

\section{References}

1. European Technical Assessment ETA-99/0009 of 06/01/2015 for Hilti HDA and HDA-R anchor

2. L. Contrafatto, R. Cosenza, Construction and Building Materials 68, 355-369 (2014)

3. P. Upadhyaya, S. Kumara, International Journal of Adhesion and Adhesives 60, 54-62 (2015)

4. J. Tistel, G. Grimstad, G. Eiksund, Journal of Rock Mechanics and Geotechnical Engineering 9, 10101030 (2017)

5. J.G.M. van Mier, Fracture Processes of Concrete (CRC Press, Boca Raton, 2000)

6. A. F. Bower, Applied mechanics of solids (CRC Press, 2009)

7. M. Elices, G.V. Guinea, J. Gómez and J. Planas, Engineering Fracture Mechanics 69, 137-163 (2002)

8. J. Podgórski, J. Gontarz, Advances in Mechanics: Theoretical, Computational and Interdisciplinary Issues, 479-483 (CRC Press, Boca Raton, 2016)

9. S. Mohammadi, Extended Finite Element Method: for Fracture Analysis of Structures (WileyBlackwell, 2007)

10. http://abaqus.software.polimi.it/v6.14/index.html

11. J. Gontarz, J. Podgórski, J. Jonak, M. Kalita, M. Siegmund, 41st Solid Mechanics Conference, Book of Abstracts, 290-291 (2018) 